\title{
Temperature-driven luminescence switching of europium(III) in a glass dispersed liquid crystal film
}

\author{
$\underline{\text { Kris Driesen and Koen Binnemans }}$ \\ K.U.Leuven, Department of Chemistry, Coordination Chemistry Division, \\ Celestijnenlaan 200F, B-3001 Leuven, BELGIUM \\ kris.driesen@chem.kuleuven.ac.be
}

Dispersed liquid crystal displays are a more recent type of LCD and involve micrometer-sized droplets of a nematic liquid crystal in a solid, isotropic matrix. ${ }^{1}$ Polymer dispersed liquid crystals are now well studied. The sol-gel process can be used for preparing films of Glass Dispersed Liquid Crystals (GDLCs). ${ }^{2}$ The glass films have dispersed liquid-crystalline droplets with a diameter varying between 1 and $180 \mu \mathrm{m}$.

Here, a simple type of GDLC was used to test the application of lanthanidedoped luminescent GDLC films. The samples were prepared by a sol-gel synthesis starting from tetraethyl orthotitanate, an amine functionalized organoalkoxysilane and water. The incorporation of a liquid crystal $(5 \mathrm{CB})$ in the sol-gel film and the solubility a europium(III) $\beta$-diketonate complex in this matrix was tested. The variation of the luminescence intensity of a europium(III) complex as a function of the temperature was recorded.

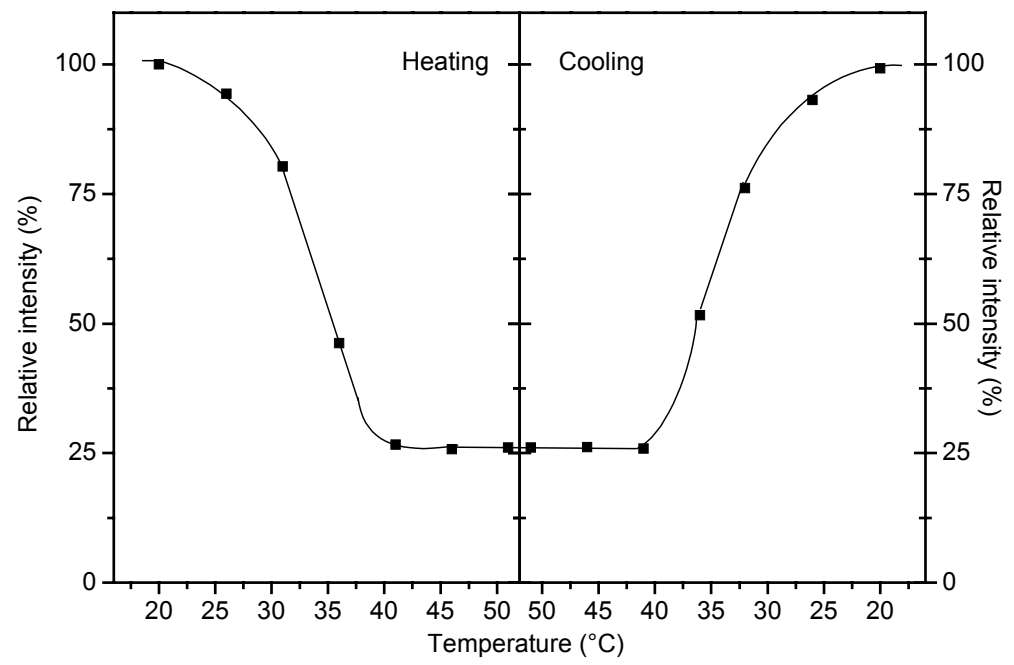

Figure 1: The relative intensity change of the ${ }^{5} D_{0} \rightarrow{ }^{7} F_{2}$ transition during heating and cooling a glass dispersed liquid crystal film doped with a europium(III) $\beta$-diketonate complex.

The liquid crystal in the nematic phase scatters incident light very strongly. In a GDLC film the droplets containing liquid crystal cause the whole sample to be turbid. UV-light was used to excite the luminescent compound. Due to the large internal scattering of the UV-light in the nematic phase the absorption by the luminescent dopand is enhanced, thus giving the layer a visible red color. When the 
film is heated above the clearing point, the refractive index of the isotropic liquid becomes similar to the refractive index of the glass. The UV-light passes through the layer with less absorption, and thus less luminescence light is produced. A strong change in the luminescence intensity was observed at the nematic-to-isotropic transition.

\section{References}

(1) Higgins, D.A., Adv. Mater. 2000, 12, 251.

(2) Levy, D., Serna, C.J., and Oton, J.M., Mater. lett. 1991, 10, 470. 